Review

\title{
Exact Solutions in Modified Gravity Models
}

\author{
Andrey N. Makarenko * and Valery V. Obukhov \\ Tomsk State Pedagogical University, 634061, Kievskaya st., 60, Tomsk, Russia \\ * Author to whom correspondence should be addressed; E-Mail: andre @ tspu.edu.ru; \\ Tel.: +7-3822-521-793.
}

Received: 24 April 2012; in revised form: 21 May 2012 / Accepted: 21 May 2012 /

Published: 25 June 2012

\begin{abstract}
We review the exact solutions in modified gravity. It is one of the main problems of mathematical physics for the gravity theory. One can obtain an exact solution if the field equations reduce to a system of ordinary differential equations. In this paper we consider a number of exact solutions obtained by the method of separation of variables. Some applications to Cosmology and BH entropy are briefly mentioned.
\end{abstract}

Keywords: modified gravity; exact solution; cosmology

\section{Introduction}

Gravitational field equations describing the geometry of space-time play a fundamental role in modern theoretical physics. Their analysis is an extremely difficult task. However, one can find an exact solution in some cases, imposing certain additional restrictions. There are several ways to impose constraints on the space-time, for example, the algebraic classification of the Weyl tensor (of Petrov types) and the Ricci tensor (Plebanski type), the choice of energy-momentum tensor from physical considerations, presence of the symmetry groups acting on a manifold, etc.

One can get the exact solution by reducing the system of equations to a system of ordinary differential equations. This can be done using the method of separation of variables. In essence, the separation of variables is the only currently known method of structural integration of the field equations. The purpose of the method consists of classification of all the privileged coordinate systems and external fields, which is a separation of variables. In the classification we refer to the transfer of all relevant space-time metrics (non-equivalent with respect to admissible coordinate transformations) satisfying the 
requirement of complete separation of variables in the equations of motion of test particles. In flat space-time classification is carried out completely.

¿From a mathematical point of view, the study of homogeneous spaces and Stäckel metrics is of special interest. The spaces are united by existence in the space of sets consisting of three geometric objects (for Stäckel spaces-Killing vector and tensor fields, for homogeneous spaces-Killing vector fields). For both cases the field equations can be reduced to a system of finite (but sufficiently large) number of ordinary differential equations. There are many methods in mathematical physics for studying such systems of equations. For example, one can use methods of additional symmetries of the system of equations, the Hamiltonian formulation of the theory of dynamical systems, etc. Essentially, all physically interesting cases (FRW cosmology, BH) belong to Stäckel and homogeneous spaces.

\section{Exact Solutions in Stäcke and Homogeneous Spaces}

Recall that metric is called the Stäckel one if the Hamilton-Jacobi equation

$$
g^{\alpha \beta} S_{, \alpha} S_{, \beta}=m^{2} \quad \alpha, \beta=1, \ldots, n
$$

can be integrated by the method of complete separation of variables. In this case the privileged co-ordinate set $u^{\alpha}$ exists for which complete integral of Equation (1) can be shown in the form

$$
S=\sum_{i=1}^{n} \phi_{i}\left(u^{i}, \lambda\right)
$$

where $\lambda_{i}$ is the essential parameter.

It appears that the other important equations of motion (Klein-Gordon-Fock, Dirac, Weyl) can be integrated by complete separation of variables only for the metrics belonging to the class of Stäckel spaces.

That is why the research of this class of spaces belongs to the one of the important problems of the mathematical physics.

The metrics of the Stäckel spaces can be used for integrating the field equations of General Relativity and other theories of Gravity. Note that such famous solutions as Schwarzschild, Kerr, NUT, Friedman and others belong to the class of Stäckel spaces. Apparently the first papers devoted to the problem of classification of the Stäckel spaces satisfying the Einstein equations were published by Carter [1]. Later in our paper [2] the complete classification of the special Stäckel electrovacuum spaces has been found. In other words, all Stäckel spaces satisfying the Einstein-Maxwell equations for the case when potentials $A_{i}$ admit complete separation of variables for Hamilton-Jacobi equation have been found. In our paper [3] the classification problem has been solved for the case when $A_{i}$ are arbitrary functions and spaces are null (types N.1). In our paper [4] all electrovacuum spacetimes admitting diagonalization and complete separation of variables for the Dirac-Fock-Ivanenko equation were found.

One of the complicated problems of the modern mathematical physics is the integration problem of the Einstein-Dirac equations.

Using spaces for which Equation (1) can be integrated by the complete separation of variables and separated solutions of the Dirac equation, one can transform Einstein-Dirac equations to the set of 
functional equations. The first papers devoted to the classification problem for the Einstein-Dirac equations were done by Bagrov, Obukhov, Sakhapov [5]. The Stäckel spaces of type (3.1) for Einstein-Dirac and Einstein-Weyl equations have been studied. Appropriate solutions have been obtained. They contain arbitrary functions depending on null variable only.

The problem of classification of Stäckel spaces for other theories of gravity for the first time was considered in papers [6-9].

We have solved the classification problem for the Einstein-Vaidya equations. Let the stress-energy tensor have the form

$$
T_{\alpha \beta}=a(x) l_{\alpha} l_{\beta}, \quad l_{\alpha} l^{\alpha}=0
$$

The solution of this problems, as well as a detailed overview of the theory of Stäckel spaces, can be found in [10].

Of high interest are homogeneous spaces, which lie at the heart of modern cosmology [11-14]. The homogeneous spaces are a base for building the Big Bang model, the initial singularity, as well as the inflationary model. It is of interest to identify the various mechanisms of isotropization of the universe [15-20]. Homogeneous spaces are also used in a variety of modern theories of gravity for the study of general regularities in the picture of the universe [21-24]. One can study effects of the gravitational field to other fields and matter on the background of homogeneous spaces [25-29].

Let us consider the Einstein-Weyl equations. One can show that for all types of Bianchi classification, a closed self-consistent system of ordinary integrable differential equations can be constructed [30,31]. For example, consider the first type of classification of Bianchi [32]. This is one of the most simple cases; to construct a general solution for all types is not possible.

All calculations will be carried out in the Newman-Penrose formalism. Einstein equation takes the form

$$
\left\{\begin{aligned}
\Phi_{00} & =G T_{000^{\prime} 0^{\prime}} \\
\Phi_{01} & =G T_{000^{\prime} 1^{\prime}} \\
\Phi_{02} & =G T_{001^{\prime} 1^{\prime}} \\
\Phi_{11} & =G T_{010^{\prime} 1^{\prime}}+\frac{G}{4} T_{A B^{\prime}}^{A B^{\prime}} \\
\Phi_{12} & =G T_{011^{\prime} 1^{\prime}} \\
\Phi_{22} & =G T_{111^{\prime} 1^{\prime}} \\
\Lambda & =\frac{1}{6} H-\frac{G}{12} T_{A B^{\prime}}^{A B^{\prime}}
\end{aligned}\right.
$$

Here $H$ is cosmological constant, $G$ is corresponds to the gravitational constant, $\Phi_{a b}$ are Ricci spinors and $T_{A B A^{\prime} B^{\prime}}$ is energy-momentum tensor,

$$
\begin{aligned}
T_{A B^{\prime} C D^{\prime}}=i k\left(\xi_{D^{\prime}} \nabla_{A B^{\prime}} \xi_{C}+\xi_{B} \nabla_{C D^{\prime}} \xi_{A}\right. & -\xi_{C} \nabla_{A B^{\prime}} \xi_{D^{\prime}}-\xi_{A} \nabla_{C D^{\prime}} \xi_{B^{\prime}}-\eta_{D^{\prime}} \nabla_{A B^{\prime}} \eta_{C} \\
& \left.-\eta_{B} \nabla_{C D^{\prime}} \eta_{A}+\eta_{C} \nabla_{A B^{\prime}} \eta_{D^{\prime}}+\eta_{A} \nabla_{C D^{\prime}} \eta_{B^{\prime}}\right)
\end{aligned}
$$

and $\nabla_{A B}$ is spinor derivative.

Ricci spinors are expressed in terms of spin factors as follows

$$
\Phi_{00}=D \rho-\bar{\delta} \kappa-\rho^{2}-\sigma \bar{\sigma}-\rho(\varepsilon+\bar{\varepsilon})+\bar{\kappa} \tau+\kappa(3 \alpha+\bar{\beta}-\pi),
$$




$$
\begin{aligned}
\Phi_{01}= & D \bar{\alpha}-\delta \bar{\varepsilon}-\bar{\alpha}(\bar{\rho}+\varepsilon-2 \bar{\varepsilon})-\bar{\beta} \sigma+\beta \bar{\varepsilon}+\bar{\kappa} \bar{\lambda}+\kappa \bar{\gamma}-\bar{\pi}(\bar{\varepsilon}+\bar{\rho}), \\
\Phi_{02}= & D \bar{\lambda}-\delta \bar{\pi}+\bar{\lambda}(3 \bar{\varepsilon}-\varepsilon-\bar{\rho})-\bar{\mu} \sigma-\bar{\pi}(\bar{\pi}-\beta+\bar{\lambda})+\bar{\nu} \kappa, \\
\Phi_{11}= & \frac{1}{2}[D \gamma-\Delta \varepsilon+\delta \alpha-\bar{\delta} \beta-\alpha(\tau+\bar{\pi})-\beta(\bar{\tau}+\pi)+\gamma(\varepsilon+\bar{\varepsilon}) \\
& +\varepsilon(\gamma+\bar{\gamma})-\tau \pi+\kappa \nu-\mu \rho+\sigma \lambda-\alpha \bar{\alpha}-\beta \bar{\beta}+2 \alpha \bar{\beta}- \\
= & \gamma(\rho-\bar{\rho})-\varepsilon(\mu-\bar{\mu})], \\
\Phi_{12}= & \delta \gamma-\Delta \beta-\gamma(\tau-\bar{\alpha}-\beta)-\mu \tau+\sigma \nu+\varepsilon \bar{\nu}+\beta(\gamma-\bar{\gamma}-\mu)-\alpha \bar{\lambda}, \\
\Phi_{22}= & \delta \nu-\Delta \mu-\mu^{2}-\lambda \bar{\lambda}-\mu(\gamma+\bar{\gamma})+\bar{\nu} \pi-\nu(\tau-3 \beta-\bar{\alpha}), \\
6 \Lambda= & 2[\bar{\delta} \tau-\Delta \rho-\rho \bar{\mu}-\sigma \lambda+\tau(\bar{\beta}-\alpha-\bar{\tau})+\rho(\gamma+\bar{\gamma}+\nu \kappa]- \\
= & D \gamma+\Delta \varepsilon+\delta \alpha-\bar{\beta}+\alpha(\tau+\bar{\pi})+\beta(\bar{\tau}+\pi)-\gamma(\varepsilon+\bar{\varepsilon})- \\
= & \varepsilon(\gamma+\bar{\gamma})+\tau \pi-\kappa \nu-\mu \rho+\lambda \sigma
\end{aligned}
$$

Choose a metric in the form

$$
g_{00}=1, \quad g_{0 \alpha}=0, \quad g_{i j}=-\gamma_{i j}
$$

where $\gamma_{i j}$ is the metric of a three-dimensional space with the signature $(+,+,+)$. It is simple to establish that this space permits a three-parameter Abelian group of motions, and hence is a Steckel space of type (3.0).

The orthogonal tetrad is chosen in the form

$$
\begin{array}{ll}
e_{(0) \alpha}=(1,0,0,0), & e_{(1) \alpha}=(0, A, B, C) \\
e_{(2) \alpha}=(0, K, S, V), & e_{(3) \alpha}=(0, P, M, Z)
\end{array}
$$

where $A, B, C, R, S, V, P, M, Z$ are arbitrary functions of the time.

Using this tetrad, we construct the Newman-Penrose tetrad

$$
\begin{aligned}
\ell_{i} & =\frac{1}{\sqrt{2}}\left(e_{(0) i}+e_{(1) i}\right), & n_{i} & =\frac{1}{\sqrt{2}}\left(e_{(0) i}-e_{(1) i}\right), \\
m_{i} & =\frac{1}{\sqrt{2}}\left(e_{(2) i}+i e_{(3) i}\right), & \overline{m_{i}} & =\frac{1}{\sqrt{2}}\left(e_{(2) i}-i e_{(3) i}\right)
\end{aligned}
$$

and obtain the following relations between the spin factors

$$
\begin{array}{ll}
\lambda=-\bar{\sigma}, \quad \nu=\bar{\kappa}, \quad \pi=\bar{\tau}, & \gamma=-\bar{\varepsilon} \\
\alpha=\bar{\beta}, \quad \mu=-\rho=\bar{\mu}, & \alpha=\frac{1}{2}(\bar{\tau}-\bar{\kappa})
\end{array}
$$

The spinor field of spatial rotation of the tetrad may be diagonalized and made real (one real component remains). For this case, the energy-momentum tensor takes the form (5).

$$
\begin{array}{lll}
T_{00^{\prime} 00^{\prime}}=2 i k a \xi_{0}^{2}(\bar{\varepsilon}-\varepsilon), & T_{00^{\prime} 01^{\prime}}=i k a \xi_{0}^{2} \tau, & T_{01^{\prime} 01^{\prime}}=-2 i k a \xi_{0}^{2} \sigma \\
T_{01^{\prime} 11^{\prime}}=i k a \xi_{0}^{2} \kappa, & T_{11^{\prime} 11^{\prime}}=0, & T_{00^{\prime} 11^{\prime}}=i k a \xi_{0}^{2}(\bar{\varepsilon}-\varepsilon), \quad T_{01^{\prime} 10^{\prime}}=0
\end{array}
$$

The field equations take the form

$$
\dot{\rho}-\rho^{2}-\sigma \bar{\sigma}-2 \rho \varepsilon-4 \kappa \bar{\kappa}=0
$$




$$
\begin{aligned}
\dot{\sigma}-2 \rho \sigma+2 \sigma \varepsilon & =2 i y \xi_{0}{ }^{2} \sigma \\
\dot{\kappa}-2 \rho \kappa+2 \kappa \varepsilon & =i y \xi_{0}{ }^{2} \kappa \\
2 \dot{\varepsilon}-\dot{\rho}+\rho^{2}+\sigma \bar{\sigma}-2 \rho \varepsilon-4 \kappa \bar{\kappa}+4 \varepsilon^{2} & =\frac{H}{2} \\
\dot{\rho}-1 \rho^{2}+2 \rho \varepsilon & =\frac{H}{2} \\
\dot{\xi}_{0}+(\varepsilon-\rho) \xi_{0} & =0
\end{aligned}
$$

where ${ }^{\cdot}=\frac{d}{d t}$.

Multiplying Equation (7) by $\bar{\sigma}$ and Equation (8) by $\bar{\kappa}$, we add and subtract the resulting equations and their conjugate forms. Transformation yields

$$
\begin{aligned}
& (\ln \dot{\kappa} \bar{\kappa})=-4(\varepsilon-\rho) \\
& (\ln \dot{\sigma} \bar{\sigma})=-4(\varepsilon-\rho)
\end{aligned}
$$

Multiplying Equation (11) by $y \xi_{0}$, we obtain

$$
\left[\ln \left(\dot{y} \xi_{0}^{2}\right)\right]=-2(\varepsilon-\rho)
$$

One find that

$$
\begin{aligned}
& \kappa=k y \xi_{0}^{2} e^{i \omega_{1}} \\
& \sigma=s y \xi_{0}^{2} e^{i \omega_{2}}
\end{aligned}
$$

Finally, we obtain the following equation

$$
(\varepsilon \dot{-} \rho)+2(\varepsilon-\rho)^{2}=\frac{3 H}{4}
$$

(1) Consider the case when $H=0$. In this case

$$
\frac{(\varepsilon-\rho)}{(\varepsilon-\rho)^{2}}=-2
$$

and thus

$$
\frac{1}{(\varepsilon-\rho)}=2 x+\text { const }
$$

Incorporating the constant into the definition of $x$, we write

$$
(\varepsilon-\rho)=\frac{1}{2 x}
$$

Hence

$$
\frac{1}{2 x}=-\frac{1}{2} \frac{\ddot{\omega}}{\dot{\omega}} \Rightarrow \quad \dot{\omega}=\frac{d}{x}
$$

where $d$ is a constant of integration. We now find $\mathrm{P}$ and $\mathrm{e}$ in explicit form

$$
\begin{gathered}
\dot{\rho}+\frac{\rho}{x}=0 \Rightarrow \rho=\frac{f}{x} \\
\varepsilon-\rho=\frac{1}{2 x} \Rightarrow \varepsilon=\frac{1}{2 x}+\frac{f}{x}=\frac{f+1 / 2}{x}
\end{gathered}
$$


where $f$ is a constant of integration. The constants of integration satisfy the condition

$$
2 f+3 f^{2}+d^{2}\left(s^{2}+4 k^{2}\right)=0
$$

Obviously, this equation has a nontrivial solution.

(2) Similarly, for the case $H \neq 0$, we can show that it is not implemented.

To find the tetrad we have to solve the two equations

$$
\begin{aligned}
& 2 A \varepsilon-(K-i P) \kappa-(K+i P) \bar{\kappa}=A^{\prime} \\
& 2 A \kappa+(K-i P) \sigma+(K+i P) \rho=-\left(K^{\prime}+i P^{\prime}\right)
\end{aligned}
$$

Since

$$
\varepsilon=\frac{1 / 2+f}{x}, \quad \rho=\frac{f}{x}, \quad \kappa=k \frac{d}{x} e^{i \omega}, \quad \sigma=s \frac{d}{x} e^{i(2 \omega+c)}
$$

they take the form

$$
\begin{aligned}
2 A \frac{1 / 2+f}{x}-(K-i P) \frac{k d}{x} e^{i \omega}-(K+i P) \frac{k d}{x} e^{-i \omega} & =A^{\prime} \\
2 A \frac{k d}{x} e^{i \omega}+(K-i P) \frac{s d}{x} e^{i(2 \omega+c)}(K+i P) \frac{f}{x} & =-\left(K^{\prime}+i P^{\prime}\right)
\end{aligned}
$$

We multiply the second equation by $e^{-i \omega}$ and make the substitution

$$
\left\{\begin{array}{l}
X=K \cos \omega+P \sin \omega \\
Y=P \cos \omega-K \sin \omega
\end{array}\right.
$$

The inverse transformations are

$$
\left\{\begin{array}{l}
K=Y \cos \omega+X \sin \omega \\
P=X \cos \omega-Y \sin \omega
\end{array}\right.
$$

We also introduce the notation $\frac{f}{d}=F, \frac{1}{d}=D$

$$
\begin{aligned}
A(2 F+D)-k(X-i Y)-k(X+i Y) & =\frac{A^{\prime}}{\omega^{\prime}} \\
2 A k+s(X-i Y) e^{i c}+F(X+i Y) & =\frac{-(X+i Y)^{\prime}-i \omega^{\prime}(X+i Y)}{\omega^{\prime}}
\end{aligned}
$$

We make one more substitution

$$
A=W e^{-F \omega}, \quad(X+i Y)=(U+i V) e^{-F \omega}
$$

and take into account that

$$
\frac{A^{\prime}}{\omega^{\prime}}=\frac{d A}{d x} \frac{d x}{d \omega}=\frac{d A}{d \omega}
$$

Then the equations take the form

$$
\begin{aligned}
W(3 F+D)-k[(U+i V)+(U-i V)] & =\frac{d W}{d \omega} \\
2 k W+s e^{i c}(U-i V) & =-\frac{d}{d \omega}(U+i V)-i(U+i V)
\end{aligned}
$$


If $e^{i c}=\alpha+i \beta, l=3 F+D$ and $\alpha^{2}+\beta^{2}=1$, then

$$
\left\{\begin{aligned}
W l-2 k U & =\frac{d W}{d \omega} \\
2 k W+c_{2} \alpha U+(s \beta-1) V & =-\frac{d U}{d \omega} \\
(s \beta+1) U-s \alpha V & =-\frac{d V}{d \omega}
\end{aligned}\right.
$$

One can obtain a third-order equation in $\mathrm{V}$

$$
\frac{d^{3} V}{d \omega^{3}}-\frac{d^{2} V}{d \omega^{2}} l-\frac{d V}{d \omega}\left(s^{2}+4 k^{2}-1\right)+V\left(4 k^{2} s \alpha+l\left(s^{2}-1\right)\right)=0
$$

The characteristic equation takes the form

$$
\lambda^{3}-\lambda^{2} l-\lambda\left(s^{2}+4 k^{2}-1\right)+\left(4 k^{2} s \alpha+l\left(s^{2}-1\right)\right)=0
$$

¿From this it is possible to find three roots $\lambda_{1}, \lambda_{2}, \lambda_{3}$

$$
\begin{gathered}
\lambda^{3}-\lambda^{2} l-\lambda\left(s^{2}+4 k^{2}-1\right)+\left(4 k^{2} s \alpha+l\left(s^{2}-1\right)\right)= \\
\lambda^{3}-\lambda^{2}\left(\lambda_{1}+\lambda_{2}+\lambda_{3}\right)-\lambda\left(\lambda_{1} \lambda_{2}+\lambda_{1} \lambda_{3}+\lambda_{2} \lambda_{3}\right)-\lambda_{1} \lambda_{2} \lambda_{3}=\left(4 k^{2} s \alpha+l\left(s^{2}-1\right)\right) \\
\left\{\begin{aligned}
\lambda_{1}+\lambda_{2}+\lambda_{3} & =l \\
\lambda_{1} \lambda_{2}+\lambda_{1} \lambda_{3}+\lambda_{2} \lambda_{3} & =-\left(s^{2}+4 k^{2}-1\right) \\
\lambda_{1} \lambda_{2} \lambda_{3} & =-\left(4 k^{2} s \alpha+l\left(s^{2}-1\right)\right)
\end{aligned}\right.
\end{gathered}
$$

In all, four cases are possible:

(1) $\lambda_{1}, \lambda_{2}, \lambda_{3}$ are pairwise unequal and real. Then

$$
V=c_{1} e^{\lambda_{1} \omega}+c_{2} e^{\lambda_{2} \omega}+c_{3} e^{\lambda_{3} \omega}
$$

(2) $\lambda_{1}$ is real, $\lambda_{2}=\overline{\lambda_{3}}$

$$
V=c_{1} e^{\lambda_{1} \omega}+c_{2} e^{\lambda_{2} \omega}+c_{3} e^{\overline{\lambda_{2}} \omega}
$$

(3) $\lambda_{1}, \lambda_{2}=\lambda_{3}$ are real

$$
V=c_{1} e^{\lambda_{1} \omega}+\left(c_{2}+c_{3} \omega\right) e^{\lambda_{2} \omega}
$$

(4) $\lambda_{1}=\lambda_{2}=\lambda_{3}=\lambda$

$$
\begin{aligned}
V & =\left(c_{1}+c_{2} \omega+c_{3} \omega^{2}\right) e^{\lambda \omega} \\
U & =\frac{-\frac{d V}{d \omega}+b \alpha V}{b \beta+1} \\
W & =\frac{\frac{d^{2} V}{d \omega^{2}}-\left(b^{2}-1\right) V}{2 a(b \beta+1)}
\end{aligned}
$$


Thus, an accurate solution of the Einstein-Weyl equation has been obtained for space-time of Bianchi type 1 . The solution only exists when $H=0$. The spin coefficients and the Weyl spinor take the form

$$
\rho=\frac{f}{x}, \quad \varepsilon=\frac{1 / 2+f}{x}, \quad \sigma=\frac{s d}{x} e^{i(2 \omega+c)}, \quad \kappa=\frac{k d}{x} e^{i \omega}, \quad y \xi_{0}^{2}=\frac{d}{x}
$$

where $f, k, s, y, c$ are constants and $\omega=d \ln x$

On integrating the equations for the tetrad, the functions obtained will depend on the form of Equation (22). As an example, the functions for the first case, when all the roots are different and real, are

$$
\begin{aligned}
& A=e^{-F \omega}\left[\frac{c_{1} e^{\lambda_{1} \omega}\left(\lambda_{1}{ }^{2}-b^{2}+1\right)+c_{2} e^{\lambda_{2} \omega}\left(\lambda_{2}{ }^{2}-b^{2}+1\right)+c_{3} e^{\lambda_{3} \omega}\left(\lambda_{3}{ }^{2}-b^{2}+1\right)}{2 a(b \beta+1)}\right] \\
& K=e^{-F \omega}\left[c_{1} e^{\lambda_{1} \omega} \frac{\left(b \alpha-\lambda_{1}\right)}{b \beta+1}+c_{2} e^{\lambda_{2} \omega} \frac{\left(b \alpha-\lambda_{2}\right)}{b \beta+1}+c_{3} e^{\lambda_{3} \omega} \frac{\left(b \alpha-\lambda_{3}\right)}{b \beta+1}\right] \\
& P=e^{-F \omega}\left[c_{1} e^{\lambda_{1} \omega}+c_{2} e^{\lambda_{2} \omega}+c_{3} e^{\lambda_{3} \omega}\right]
\end{aligned}
$$

where $\lambda_{1}, \lambda_{2}, \lambda_{3}$ are the roots of Equation (22); F, $a, b, c_{1}, c_{2}, c_{3}, \alpha, \beta$ are constant.

The other functions specifying the tetrad are of analogous form. The only difference is that another set of arbitrary constants must be chosen in place of $c_{1}, c_{2}, c_{3}$.

\section{Modified Gravity}

In the previous sections a few exact solutions of the classical theory of gravitation were obtained. However, in recent years, modified gravity theory is very popular. These theories my be studied also using the methods described above. Typically, solutions with a spatially flat metric depend only on time. It corresponds to the first type of Bianchi and type (3.1) on the classification of Stäckel.

The most popular models are models of modified gravity [33-35], which represents a classical generalization of general relativity (modifications of the Hilbert-Einstein action by introducing different functions of the Ricci scalar [34-38] or Gauss-Bonnet invariant [39-45]), should consistently describe the early-time inflation and late-time acceleration, without the introduction of any other dark component.

In the framework of these theories, a number of cosmological models have been constructed to adequately describe the current observational data [46-51]. In addition, under this theory there may be objects such as black holes. The properties of these objects are different from classical ones. For example the black hole entropy in the model F (R) gravity will have the form $[52,53]$

$$
S=\frac{A_{H}}{4} f^{\prime}\left(R_{0}\right)
$$

where $A_{H}=4 \pi r_{H}^{2}$.

As an example of the exact solution in modified theory of gravity. We consider the sixth dimensional Gauss-Bonnet theory [54,55].

We shall start from the following string-inspired action in six dimensions

$$
S=\int d^{6} x \sqrt{-g}\left(R+\epsilon L_{G B}\right)
$$


where $\epsilon$ is a constant and $L_{G B}$ is Gauss-Bonnet invariant

$$
L_{G B}=R_{\mu \nu \alpha \beta} R^{\nu \mu \alpha \beta}-4 R_{\mu \nu} R^{\mu \nu}+R^{2}
$$

The metric is the product of the usual metric corresponding to the 4-dimensional FRW universe and a 2-dimensional surface, namely

$$
d s^{2}=-d t^{2}+a^{2}(t)\left[\left(d x^{1}\right)^{2}+\left(d x^{2}\right)^{2}+\left(d x^{3}\right)^{2}\right]+b^{2}(t)\left[\left(d x^{4}\right)^{2}+\left(d x^{5}\right)^{2}\right],
$$

the scalar curvature is

$$
R=\frac{6 \dot{a}^{2}}{a^{2}}+\frac{12 \dot{a} \dot{b}}{a b}+\frac{2 \dot{b}^{2}}{b^{2}}+\frac{6 \ddot{a}}{a}+\frac{4 \ddot{b}}{b}
$$

while the four-dimensional and topologically invariant Gauss-Bonnet Lagrangian, $L_{G B}$, has the form

$$
L_{G B}=\frac{48 \dot{a}^{3} \dot{b}}{a^{3} b}+\frac{72 \dot{a}^{2} \dot{b}^{2}}{a^{2} b^{2}}+\frac{24 \dot{a}^{2} \ddot{a}}{a^{3}}+\frac{96 \dot{a} \ddot{a} \dot{b}}{a^{2} b}+\frac{24 \ddot{a} \dot{b}^{2}}{a b^{2}}+\frac{48 \dot{a}^{2} \ddot{b}}{a^{2} b}+\frac{48 \dot{a} \ddot{b} \ddot{b}}{a b^{2}}
$$

or, equivalently,

$$
L_{G B}=\frac{24}{a^{3} b^{2}}\left(2 \dot{a}^{3} b \dot{b}+3 a \dot{a}^{2} \dot{b}^{2}+\dot{a}^{2} \ddot{a} b^{2}+4 a \dot{a} \ddot{a} b \dot{b}+a^{2} \ddot{a} \dot{b}^{2}+2 a \dot{a}^{2} b \ddot{b}+2 a^{2} \dot{a} \dot{b} \ddot{b}\right)
$$

The corresponding equations of motion are obtained by variation of the action with respect to $a$ and $b$, which yields

$$
\begin{aligned}
\dot{a}^{2} b^{2}+4 a \dot{a} b \dot{b}+a^{2} \dot{b}^{2}+2 a \ddot{a} b^{2}+2 a^{2} b \ddot{b}+12 \epsilon \dot{a}^{2} \dot{b}^{2}+16 \epsilon \dot{a} \ddot{a} b \dot{b}+8 \epsilon a \ddot{a} \dot{b}^{2}+8 \epsilon \dot{a}^{2} b \ddot{b}+16 \epsilon a \dot{a} \dot{b} \ddot{b}=0 \\
3 a \dot{a}^{2} b+3 a^{2} \dot{a} \dot{b}+3 a^{2} \ddot{a} b+a^{3} \ddot{b}+12 \epsilon \dot{a}^{3} \dot{b}+12 \epsilon \dot{a}^{2} \ddot{a} b+24 \epsilon a \dot{a} \ddot{a} \dot{b}+12 \epsilon a \dot{a}^{2} \ddot{b}=0
\end{aligned}
$$

These equations can be easily rewritten in terms of the Hubble rates $H=a^{\prime} / a$ and $h=b^{\prime} / b$, namely

$$
\begin{aligned}
& 3 h^{2}+4 h H+3 H^{2}+ \dot{h}+2 \dot{H}+16 \epsilon h^{3} H+28 \epsilon h^{2} H^{2}+16 \epsilon h H^{3} \\
&+16 \epsilon h \dot{h} H+8 \epsilon \dot{h} H^{2}+8 \epsilon h^{2} \dot{H}+16 \epsilon h H \dot{H}=0 \\
& h^{2}+3 h H+6 H^{2}+\dot{h}+3 \dot{H}+12 \epsilon h^{2} H^{2}+36 \epsilon h H^{3}+12 \epsilon H^{4} \\
&+12 \epsilon \dot{h} H^{2}+24 \epsilon h H \dot{H}+12 \epsilon H^{2} \dot{H}=0
\end{aligned}
$$

In addition, variation over the metric in the above expressions gives the constraint equation

$$
h^{2}+6 h H+3 H^{2}+36 \epsilon h^{2} H^{2}+24 \epsilon h H^{3}=0
$$

This equation helps to exclude $h$ and $h^{\prime}$ from Equation (30). As a result, one gets an equation for $H$ only:

$$
H^{\prime}=3 H^{2} \times \frac{\sqrt{6}+4 G+\epsilon(-22 \sqrt{6}+64 G) H^{2}-24 \epsilon^{2}(9 \sqrt{6}-52 G) H^{4}+96 \epsilon^{3}(17 \sqrt{6}+12 G) H^{6}-8064 \sqrt{6} \epsilon^{4} H^{8}}{\sqrt{6}-12 \epsilon(\sqrt{6}+16 G) H^{2}+72 \epsilon^{2}(3 \sqrt{6}-32 G) H^{4}-2880 \sqrt{6} \epsilon^{3} H^{6}+31104 \sqrt{6} \epsilon^{4} H^{8}}
$$

where

$$
G=\sqrt{1-6 \epsilon H^{2}+24 \epsilon^{2} H^{4}}
$$

One can check that this last equation obeys the fundamental relation (for $\epsilon>0$ ):

$$
H^{\prime}=\frac{H^{2}\left(H^{2}-p^{2}\right)}{\left(H^{2}-q^{2}\right)\left(H^{2}-r^{2}\right)} f(H)
$$


where $p, q$ and $r$ are constants, and the function $f(H)<0$.

For $\epsilon=0$ one does recover (as it should be) an explicit solution. In other cases, only a numerical analysis can be carried out. In fact, the equations for $a, b$ are

$$
\begin{aligned}
b^{2} a^{\prime 2}+4 a b a^{\prime} b^{\prime}+a^{2} b^{\prime 2}+2 a b^{2} a^{\prime \prime}+2 a^{2} b b^{\prime \prime} & =0 \\
3 a b a^{\prime 2}+3 a^{2} a^{\prime} b^{\prime}+3 a^{2} b a^{\prime \prime}+a^{3} b^{\prime \prime} & =0
\end{aligned}
$$

and, from here,

$$
\begin{aligned}
3 h^{2}+4 h H+3 H^{2}+2 h^{\prime}+2 H^{\prime} & =0 \\
h^{2}+3 h H+6 H^{2}+h^{\prime}+3 H^{\prime} & =0
\end{aligned}
$$

from where one gets that

$$
H^{\prime}=(3 \pm 2 \sqrt{6}) H^{2}
$$

and the solution is given by

$$
H=-\frac{1}{\alpha t+C_{1}}
$$

being

$$
\alpha=3 \pm 2 \sqrt{6}
$$

Moreover, in terms of the scale factors:

$$
\begin{aligned}
& a=C_{2}\left[ \pm\left(\alpha t+C_{1}\right)\right]^{-1 / \alpha} \\
& b=C_{3}\left[ \pm\left(\alpha t+C_{1}\right)\right]^{\beta / \alpha}
\end{aligned}
$$

where

$$
\beta=3 \pm \sqrt{6}
$$

General approach to Stäckel spaces of first sections may be applied to such theory as well. However, this is more complicated in modified gravity.

\section{Conclusions}

In this article we consider the exact solutions constructed in the classical theory of Stäckel spaces and partially homogeneous spaces. We consider in detail the exact solution of Einstein-Weyl space of the first type of classification of Bianchi in the Newman-Penrose formalism. As an example we consider the exact solution for the modified gravity theory of the Gauss-Bonnet for the six-dimensional metric which depends only on time and has a diagonal form. Thus, we discuss the problem of obtaining exact solutions in the different theories of gravity. The paper shows how, by use of the method of separation of variables, can one construct exact solutions of cosmological models, both for space with matter (the classical theory of gravity) and in the case of the modified theories of gravity (six-dimensional theory of the Gauss-Bonnet). 
Note that separation of field equations for modified gravity in Stäckel space may be done in analogy with the method developed at the beginning of this work. However, the corresponding generalization is very cumbersome technically.

\section{References}

1. Carter, B. Hamilton-Jacobi and Schrodinger separable solutions of Einsteins equations. Commum. Math. Phys. 1968, 10, 280-310.

2. Bagrov, V.G.; Obukhov, V.V.; Shapovalov, A.V. Special Stäckel electrovac space times. Pramana J. Phys. 1986, 26, 93-98.

3. Bagrov, V.G.; Obukhov, V.V.; Osetrin, K.E. Classification of null-Stäckel electrovac metrics with cosmological constant. Gen. Relat. Gravit. 1988, 20, 1141-1154.

4. Bagrov, V.G.; Obukhov, V.V. New method of integration for the Dirac equation on a curved space-time. J. Math. Phys. 1992, 33, 2279-2289.

5. Bagrov, V.G.; Obukhov, V.V.; Sakhapov, A.G. Integration of the Einstein-Dirac equations. J. Math. Phys. 1996, 37, 5599-5610.

6. Bagrov, V.G.; Obukhov, V.V. Exact solutions of vacuum Brans-Dicke equations. Sov. Phys. J. 1992, $35,70-73$.

7. Bagrov, V.G.; Obukhov, V.V. Accurate integration of scalar equations in multiscalar-tensor theory. Russ. Phys. J. 1995, 38, 171-176.

8. Obukhov, V.V. Cosmological solution in the scalar-tensor theory of gravity. Russ. Phys. J. 1997, 40, 112-115.

9. Obukhov, V.V. Exact cosmological solutions of field equations of a tensor-multiscalar theory. Grav. Cosmol. 1996, 2, 256-258.

10. Obukhov, V.V. The Stäckel Spaces in Gravity Theory; Tomsk State Pedagogical Uviversity Press: Tomsk, Russia, 2006.

11. Friedman, A. Über die Krümmung des Raumes. Zs Phys. 1922, 10, 377-380.

12. Friedman, A. Über die Krümmung des Raumes. Zs Phys. 1924, 21, 326-332.

13. Taub, A.H. Empty space-times admitting a three parameter group of motions. Ann. Math. 1951, 53, 472-490.

14. Ryan, M.; Shepley, L. Homogeneous Relativistic Cosmologies; Princeton University Press: Princeton, NJ, USA, 1975.

15. Chauvet, P.; Cervantes-Cota, J.L. Isotropization of bianchi-type cosmological solutions in bransdicke theory Phys. Rev. D 1995, 52, 3416-3423.

16. Chiba, T.; Mukohyama, S.; Nakamura, T. Anisotropy of the cosmic background radiation implies the violation of the strong energy condition in bianchi type I universe. Phys. Lett. B 1997, 408, 47-51.

17. Bergamini, R.; Sedici, P.; Verrocchio, P. Inflation for Bianchi IX model. Phys. Rev. D 1997, 55, 1896-1900.

18. Byland, S.; Scialom, D. Evolution of the Bianchi I, the Bianchi III and the Kantowski-Sachs Universe: Isotropization and Inflation Phys.Rev. D. 1998, 57, 6065-6074. 
19. Nojiri, S.; Obregon, O.; Odintsov, S.D.; Osetrin, K.E. Can primordial wormholes be induced by GUTs at the early universe? Phys. Lett. B 1999, 458, 19-28.

20. Cervantes-Cota, J. L.; Nahmad, M. Isotropization of Bianchi type models and a new FRW solution in Brans-Dicke theory. Gen. Relat. Gravit. 2001, 33, 767-780.

21. Aguirregabiria, J.M.; Feinstein, A.; Ibanez, J. Exponential-potential scalar field universes I: The Bianchi I models. Phys. Rev. D 1993, 48, 4662-4668.

22. Cheng, A.D.Y.; D'Eath, P.D. Diagonal quantum Bianchi type IX models in $N=1$ supergravity. Class. Quantum Grav. 1996, 13, 3151-3162.

23. Randall, L.; Sundrum, R. An Alternatine to Compactification. Phys. Rev. Lett. 1999, 83, 46904693.

24. Csaki, C.; Joshu, E.; Grosean, C. Gravitational lorentz violations and adjustment of the cosmological constant in asymmetrically warped space-times. Nucl. Phys. B 2001, 604, 312-342.

25. Barut, A.O.; Duru, I.H. Exact solutions of the Dirac equation in spatially flat Robertson-Walker spase-times. J. Math. Phys. 1987, 36, 3705-3711.

26. Kovalyov, M.; Legare, M. The Dirac equation in Robertson-Walker spaces: A class of solutions. $J$. Math. Phys. 1990, 31, 191-198.

27. Villalba, V.H.; Percoco, U. Separation of variables and exact solution of Dirac and Weyl equations in Robertson-Walker space-times. J. Math. Phys. 1990, 31, 715-720.

28. Note Guello, E.A.; Capelas de Oliveira, E. Klein-Gordon and Dirac equations in deSitter spacetime. Int. J. Theor. Phys. 1990, 38, 585-598.

29. Gavrilov, S.P.; Gitman, D.M.; Goncalves, A.E. Quantum spinor fields in FRW Universe with a constant electromagnetic background. Int. J. Mod. Phys. A 1997, 12, 4837-4868.

30. Makarenko, A.N.; Obukhov, V.V., Osetrin, K.E. Integrability of Einstein-Weyl equations for spatially homogeneous models of type III by Bianchi. Russ. Phys. J. 2002, 45, 49-55.

31. Bagrov, V.G.; Makarenko, A.N.; Obukhov, V.V., Osetrin, K.E. Spinor fields in homogeneous cosmological models. Gravit. Cosmol. Suppl. 2002, 8, 3-5.

32. Makarenko, A.N.; Obukhov, V.V. Cosmological solution of the Einstein-Weyl equation. Russ. Phys. J. 1998, 41, 1124-1133.

33. Sahni, V.; Starobinsky, A. Reconstructing dark energy. Int. J. Mod. Phys. D 2006, 15, 2105-2132.

34. Nojiri, S.; Odintsov, S.D. Introduction to modified gravity and gravitational alternative for dark energy. Int. J. Geom. Meth. Mod. Phys. 2007, 4, 115-146.

35. Nojiri, S.; Odintsov, S.D. Unified cosmic history in modified gravity: From $F(R)$ theory to Lorentz non-invariant models. Phys. Rep. 2011, 505, 59-144.

36. Capozziello, S.; Francaviglia, M. Extended theories of gravity and their cosmological and astrophysical applications. Gen. Relat. Gravit. 2008, 40, 357-420.

37. Capozziello, S.; Faraoni, V. Beyond Einstein Gravity: A Survey of Gravitational Theories for Cosmology and Astrophysics; Springer: Berlin, Heidelberg, Germany, 2010.

38. Lobo, F.S.N. The dark side of gravity: Modified theories of gravity. 2008, arXiv:0807.1640.

39. Sami, M.; Toporensky, A.; Tretjakov, P.V.; Tsujikawa, S. The fate of (phantom) dark energy universe with string curvature corrections. Phys. Lett. B 2005, 619, 193-200. 
40. Alimohammadi, M.; Ghalee, A. Remarks on generalized Gauss-Bonnet dark energy. Phys. Rev. D 2009, 79, 063006:1-063006:7.

41. de Felice, A.; Tsujikawa, S. Solar system constraints on $\mathrm{f}(\mathrm{G})$ gravity models. Phys. Rev. D 2009, 80, 063516:1-063516:15.

42. Nojiri, S.; Odintsov, S.D. Modified Gauss-Bonnet theory as gravitational alternative for dark energy. Phys. Lett. B 2005, 631, 1-6.

43. Nojiri, S.; Odintsov, S.D.; Sasaki, M. Gauss-Bonnet dark energy. Phys. Rev. D 2005, 71, 123509:1 $-123509: 7$.

44. Myrzakulov, R.; Saez-Gomez, D.; Tureanu, A. On the $\Lambda$ CDM Universe in $f(G)$ gravity. Gen. Rel. Grav. 2011, 43, 1671-1684.

45. Elizalde, E.; Myrzakulov, R.; Obukhov, V.V.; Saez-Gomez, D. LambdaCDM epoch reconstruction from $\mathrm{F}(\mathrm{R}, \mathrm{G})$ and modified Gauss-Bonnet gravities. Class. Quant. Grav. 2010, 27, 095007:1095007:14.

46. Riess, A.G.; Filippenko, A.V.; Challis, P.; Clocchiatti, A.; Diercks, A.; Garnavich, P.M.; Gilliland, R.L.; Hogan, C.J.; Jha, S.; Kirshner, R.P.; et al. Observational evidence from supernovae for an accelerating universe and a cosmological constant. Astron. J. 1998, 116, 1009-1038.

47. Riess, A.G.; Kirshner, R.P.; Schmidt, B.P.; Jha, S.; Challis, P.; Garnavich, P.M.; Esin, A.A.; Carpenter, C.; Grashius, R.; Schild, R.E.; et al. BV RI light curves for 22 type Ia supernovae. Astron. J. 1999, 117, 707-724.

48. Perlmutter, S.; Aldering, G.; Valle, M.D.; Deustua, S.; Ellis, R.S.; Fabbro, S.; Fruchter, A.; Goldhaber, G.; Groom, D.E.; Hook, I.M.; et al. Discovery of a supernova explosion at half the age of the universe and its cosmological implications. Nature 1998, 391, 51-54.

49. Hicken, M.; Wood-Vasey, W.M.; Blondin, S.; Challis, P.; Jha, S.; Kelly, P.L.; Rest, A.; Kirshner, R.P. Improved dark energy constraints from 100 new cfa supernova type ia light curves. Astrophys. J. 2009, 700, 1097-1140.

50. Dunkley, J.; Spergel, D.N.; Komatsu, E.; Hinshaw, G.; Larson, D.; Nolta, M.R.; Odegard, N.; Page, L.; Bennett, C.L.; Gold, B.; et al. Five-Year Wilkinson Microwave Anisotropy Probe (WMAP) observations: Cosmological interpretation. Astrophys. J. Suppl. 2009, 180, 330-376.

51. Percival, W.J.; Beth A. Reid, ; Eisenstein, D.J.; Bahcall, N.A.; Budavari, T.; Frieman, J.A.; Fukugita, M.; Gunn, J.E.; Ivezic, Z.; Knapp, G.R.; et al. Baryon acoustic oscillations in the sloan digital sky survey data release 7 galaxy sample. Mon. Not. Roy. Astron. Soc. 2010, 401, $2148-2168$.

52. Cognola, G.; Elizalde, E.; Nojiri, S.; Odintsov, S.D.; Zerbini, S. One-loop f(R) gravity in de Sitter universe. JCAP 2005, 2005, doi:10.1088/1475-7516/2005/02/010.

53. Sebastiani, L.; Zerbini, S. Static spherically symmetric solutions in $\mathrm{f}(\mathrm{r})$ gravity. Eur. Phys. J. C 2011, 71, 1591:1-1591:8.

54. Elizalde, E.; Makarenko, A.N.; Obukhov, V.V.; Osetrin, K.E.; Filippov, A.E. Stationary vs. singular points in an accelerating FRW cosmology derived from six-dimensional Einstein-Gauss-Bonnet gravity. Phys. Lett. B 2007, 644, 1-6. 
55. Kirnos, I.V.; Makarenko, A.N. Accelerating cosmologies in Lovelock gravity with dilaton. Open Astron.J. 2010, 3, 37-48.

(c) 2012 by the authors; licensee MDPI, Basel, Switzerland. This article is an open access article distributed under the terms and conditions of the Creative Commons Attribution license (http://creativecommons.org/licenses/by/3.0/). 